\title{
Baysian and NonBaysian Methods to Estimate the two parameters of Logistic Distribution
}

\author{
IdenH. Alkanani* \\ Sara S. Taher**
}

Received 25, June, 2013

Accepted 3, November, 2013

\begin{abstract}
:
In this paper ,the problem of point estimation for the two parameters of logistic distribution has been investigated using simulation technique. The rank sampling set estimator method which is one of the Non_Baysian procedure and Lindley approximation estimator method which is one of the Baysian method were used to estimate the parameters of logistic distribution. Comparing between these two mentioned methods by employing mean square error measure and mean absolute percentage error measure .At last simulation technique used to generate many number of samples sizes to compare between these methods.
\end{abstract}

Keywords: logistic distribution, Lindley approximation method, Rank Sampling Set Method,Mean square error, Mean absolute percentage error , simulation technique .

\section{Introduction:}

The logistic function is one of the most popular and widely used for growth models in demographic studies and proposed by Verhulst's(18381845)[1].The normal distribution resembles to logistic distribution in shape but the logistic distribution has thicker tails and higher kurtosis than normal distribution.

Plackett (1959) used the logistic function in the analysis of survival data[2] ;Oliver (1964) used the logistic function as a model for agricultural production data[3];Henrick .and Bovas(1973) suggested two families of multivariate logistic distributions[4] ;Balakrishnan and leung (1988) clarify the probability density function of type I generalized logistic distribution which defined by the following formula[5] :

$f(x ; b)=\frac{b e^{-x}}{\left(1+e^{-x}\right)^{b+1}}-\infty<\mathrm{x}<\infty \quad \mathrm{b}>0$

Scerri and Farrugia(1996) compared between the logistic distribution and weibull distribution for modeling wind speed data[6]; Jones (2006)find out that logistic distribution is a special case of class function[7] :

$f(x)=f(x ; \alpha, \beta)=F(x)^{\alpha}(1-$
$F(x))^{\beta} \quad-\infty<\alpha<\infty \quad \beta>0$

ZahraaA.A(2009); estimated the parameters of generalized logistic distribution by using four method such as(moment method, maximum likelihood method, modified moment method and least squares method) by utilizing two procedures of Monte carlo simulation for generating random variables from the logistic distribution[8]; Subrata ,Partha and Mosoom(2011) proposed askew logistic distribution then they derived some properties for this distribution[9]. The random variable $(X)$ has logistic distribution which contain two parameters, it has the following cummlative distribution function (cdf):

$F(X ; \alpha, \beta)=\frac{1}{1+e^{-(x-\alpha) / \beta}}(1)$

Where $\alpha$ is location parameter and $\beta$ is scale parameter .The probability density function (pdf) for the logistic distribution defined as follows:

*Department of Mathematics, College of Science for Women, University of Baghdad, Baghdad, Iraq.

**Department of Mathematics, College of Education For Pure Science /IbnAL_Haitham, University of

Baghdad, Baghdad, Iraq. 


$$
f(x ; \alpha, \beta)=\left\{\begin{array}{cc}
\frac{e^{-\frac{x-\alpha}{\beta}}}{\beta\left[1+e^{-\frac{x-\alpha}{\beta}}\right]^{2}} & -\infty<x<\infty,-\infty<\alpha<\infty, \beta>0 \\
0 & \text { o.w }
\end{array}\right.
$$

The mean ,variance for this distribution define as follows:

$M_{x}=\alpha \sigma_{x}^{2}=\frac{\pi^{2}}{3}$

Lindley Approximation Estimator Method (LAE):-

Lindley approximation method proposed by D.V.lindley in (1980)[10]; the procedure was designed and used to obtain the approximate Bayes estimators, and compute the ratio of two integrals as follows:-

$\frac{\int \mathrm{w}(\theta) \mathrm{e}^{\mathrm{L}(\theta)} \partial \theta}{\int \mathrm{v}(\theta) \mathrm{e}^{\mathrm{L}(\theta)} \partial \theta}(3)$

Where $\theta=\left(\theta_{1}, \theta_{2} \ldots \ldots \theta_{n}\right)$ are a parameters

$L(\theta)=\sum_{i=1}^{n} \ln f\left(x_{i} / \theta\right)$ is the logarithm Likelihood function for $\mathrm{n}$ observation

where $\mathrm{w}(\theta), \mathrm{V}(\theta)$ are any spot function for parameters.

$f(\alpha)=\left\{\begin{array}{lr}c e^{-\alpha c} & \alpha>0 \\ 0 & \text { o. } w\end{array}\right.$

$f(\beta)= \begin{cases}\frac{a^{n}}{\Gamma(n)} \beta^{n-1} e^{-\beta a} & \beta>0 \\ 0 & \text { o.w }\end{cases}$

The likelihood function of two - parameters logistic distribution is:-

$$
\begin{aligned}
& L\left(x_{1}, x_{2} \ldots \ldots x_{n} ; \alpha, \beta\right)=\prod_{i=1}^{n} f\left(x_{i} ; \alpha, \beta\right) \\
& L\left(x_{1}, x_{2} \ldots \ldots x_{n} ; \alpha, \beta\right)=\prod_{i=1}^{n}\left[\frac{e^{-\left(x_{i}-\alpha\right) / \beta}}{\beta\left[1+e^{-\left(x_{i}-\alpha\right) / \beta}\right]^{2}}\right] \\
& L=\frac{e^{-\sum_{i=1}^{n}\left(x_{i-} \alpha\right) / \beta}}{\beta^{n} \prod_{i=1}^{n}\left[1+e^{-\left(x_{i}-\alpha\right) / \beta}\right]^{2}}
\end{aligned}
$$

The joint p.d.f of $\alpha, \beta$ is : -

$$
\begin{aligned}
& J\left(x_{1}, x_{2} \ldots \ldots x_{n} ; \alpha, \beta\right)=l\left(x_{1}, x_{2} \ldots \ldots x_{n} ; \alpha, \beta\right) f(\alpha) f(\beta) \\
& J\left(x_{1}, x_{2} \ldots \ldots x_{n} ; \alpha, \beta\right)=\frac{e^{-\sum_{i=1}^{n}\left(x_{i}-\alpha\right) / \beta}}{\beta^{n} \prod_{i=1}^{n}\left[1+e^{-\left(x_{i}-\alpha\right) / \beta}\right]^{2}} \frac{a^{n}}{\Gamma(n)} c \beta^{n-1} e^{-(\beta a+c \alpha)}
\end{aligned}
$$


The posterior density function of $\alpha$ and $\beta$ is :-

$$
\begin{aligned}
R\left(\alpha, \beta / x_{1}, x_{2} \ldots \ldots x_{n}\right)= & \frac{l\left(x_{1}, x_{2} \ldots \ldots x_{n} ; \alpha, \beta\right) f(\alpha) f(\beta)}{\iint_{0}^{\infty} l\left(x_{1}, x_{2} \ldots \ldots x_{n} ; \alpha, \beta\right) f(\alpha) f(\beta) \partial \alpha \partial \beta} \\
R\left(\alpha, \beta / x_{1}, x_{2} \ldots \ldots x_{n}\right)= & \frac{\frac{e^{-\sum_{i=1}^{n}\left(x_{i}^{-\alpha}\right) / \beta}}{\beta^{n} \Gamma(n) \prod_{i=1}^{n}\left[1+e^{-\left(x_{i}-\alpha\right) / \beta}\right]^{2}} c a^{n} \beta^{n-1} e^{-(\beta a+c \alpha)}}{\iint_{0}^{\infty} \frac{e^{-\sum_{i=1}^{n}-\left(x_{i}-\alpha\right) / \beta}}{\Gamma(n) \beta^{n} \prod_{i=1}^{n}\left[1+e^{-\left(x_{i}-\alpha\right) / \beta}\right]^{2}} c a^{n} \beta^{n-1} e^{-(\beta a+c \alpha)} \partial \alpha \partial \beta}
\end{aligned}
$$

The Baysian estimator for $\alpha$ and $\beta$ by using squared error loss function is :$\hat{R}=E[R(\alpha, \beta)]$

Where $\mathrm{R}(\alpha, \beta)$ be any function, for $\alpha$ and $\beta$ :-

$$
\begin{gathered}
\hat{R}=E[R(\alpha, \beta)]=\frac{\iint_{0}^{\infty} R(\alpha, \beta) l\left(x_{1}, x_{2} \ldots \ldots x_{n} ; \alpha, \beta\right) f(\alpha) f(\beta) \partial \alpha \partial \beta}{\iint_{0}^{\infty} l\left(x_{1}, x_{2} \ldots \ldots x_{n} ; \alpha, \beta\right) f(\alpha) f(\beta) \partial \alpha \partial \beta} \\
\hat{R}=\frac{\iint_{0}^{\infty} \frac{e^{-\sum_{i=1}^{n}\left(x_{i}-\alpha\right) / \beta}}{\beta^{n} \Gamma(n) \prod_{i=1}^{n}\left[1+e^{-\left(x_{i}^{-\alpha}\right) / \beta}\right]^{2}} c a^{n} \beta^{n-1} e^{-(\beta a+c \alpha)} R(\alpha, \beta) \partial \alpha \partial \beta}{\iint_{0}^{\infty} \frac{e^{-\sum_{i=1}^{n}\left(x_{i}-\alpha\right) / \beta}}{\beta^{n} \Gamma(n) \prod_{i=1}^{n}\left[1+e^{-\left(x_{i}-\alpha\right) / \beta}\right]^{2}} c a^{n} \beta^{n-1} e^{-(\beta a+c \alpha)} \partial \alpha \partial \beta}
\end{gathered}
$$

By using lindely's approximate which approximate the ratio of the two integrals $\hat{R}$ to get Bayes estimators approximation as follows in the next few steps :-

The Bayes estimators can be written as follows :-

$\hat{R}=R(\hat{\alpha}, \hat{\beta})+\frac{1}{2}\left[A+l_{30} \beta_{12}+l_{03} \beta_{21}+l_{21} C_{12}+l_{12} C_{21}\right]+p_{1} A_{12}+p_{2} A_{21}$

Where $A=\sum_{i=1}^{n} \sum_{j=1}^{n} W_{i j} T_{i j} \quad ; i+j=3$

And $\quad l_{i j}=\frac{\partial^{i+j} \ln L(\alpha, \beta)}{\partial \alpha^{i} \partial \beta^{j}}$

$$
\begin{aligned}
& p_{i}=\frac{\partial p}{\partial \beta_{i}} \\
& W_{1}=\frac{\partial R}{\partial \alpha} \\
& W_{2}=\frac{\partial R}{\partial \beta}
\end{aligned}
$$

$$
p=\ln f(\alpha, \beta)
$$

$\beta_{i j}=\left(W_{i} T_{i j}+W_{j} T_{i j}\right) T_{i i} C_{i j}=3 W_{i} T_{i i} T_{i j}+W_{i}\left(T_{i i} T_{j j}+2 T^{2}{ }_{i j}\right)$

We must taking the log-likelihood function of logistic distribution:

Now;

$$
\ln L\left(x_{i} ; \alpha, \beta\right)=-n \ln \beta-\frac{1}{\beta} \sum_{i=1}^{n}\left(x_{i}-\alpha\right)-2 \sum_{i=1}^{n} \ln [1+
$$

$\left.e^{-\left(x_{i}-\alpha\right) / \beta}\right]$

To find $l_{12}=\frac{\partial^{3} \operatorname{Ln} L\left(x_{i} ; \alpha, \beta\right)}{\partial \alpha \partial \beta^{2}}$ we apply the following formulas: 
$\frac{\partial \operatorname{Ln} L}{\partial \alpha}=\frac{n}{\beta}-\frac{2}{\beta} \sum_{i=1}^{n} \frac{e^{-\left(x_{i}-\alpha\right) / \beta}}{1+e^{-\left(x_{i}-\alpha\right) / \beta}}$

$\frac{\partial^{2} L n L}{\partial \alpha \partial \beta}=\frac{-n}{\beta^{2}}-2 \sum_{i=1}^{n} \frac{e^{-\left(x_{i}-\alpha\right) / \beta}\left[\frac{x_{i}-\alpha}{\beta}-1-e^{-\left(x_{i}-\alpha\right) / \beta}\right]}{\left[\beta+\beta e^{-\left(x_{i}-\alpha\right) / \beta}\right]^{2}}$

$\frac{\partial^{3} L n L}{\partial \alpha \partial \beta^{2}}=\frac{n}{\beta^{3}}-\frac{1}{\beta^{3}} \sum_{i=1}^{n} \frac{\frac{\left(x_{i}-\alpha\right)^{2}}{\beta^{2}} e^{-\left(x_{i}-\alpha\right) / \beta}-\frac{4\left(x_{i}-\alpha\right)}{\beta} e^{-(\mathrm{xi}-\alpha) / \beta}-\frac{\left(x_{i}-\alpha\right)^{2}}{\beta^{2}} e^{-2(\mathrm{xi}-\alpha) / \beta}}{\left[1+e^{-\left(x_{i}-\alpha\right) / \beta}\right]^{3}}$

$+\frac{2 \mathrm{e}^{-2\left(x_{i}-\alpha\right) / \beta}+4 \mathrm{e}^{-2\left(x_{i}-\alpha\right) / \beta}+2 \mathrm{e}^{-3\left(x_{i}-\alpha\right) / \beta}}{\left[1+\mathrm{e}^{-\left(x_{i}^{-\alpha}\right) / \beta}\right]^{3}}$

Therefore, we find $l_{21}=\frac{\partial^{3} L n L}{\partial \alpha^{2} \partial \beta}$ by following equation:

$\frac{\partial^{2} \operatorname{LnL}}{\partial \alpha^{2}}=\frac{-2}{\beta^{2}} \sum_{i=1}^{n} \frac{e^{-\left(x_{i}-\alpha\right) / \beta}}{\left[1+e^{-\left(x_{i}-\alpha\right) / \beta}\right]^{2}}$

$\frac{\partial^{3} L n L}{\partial \alpha^{2} \partial \beta}=\frac{-2}{\beta^{3}} \sum_{i=1}^{n} \frac{e^{-\left(x_{i}^{-\alpha}\right) / \beta}\left[\frac{x_{i}-\alpha}{\beta}-\frac{x_{i}-\alpha}{\beta} e^{-\left(x_{i}-\alpha\right) / \beta}-2 e^{-\left(x_{i}-\alpha\right) / \beta}-2\right]}{\left[1+e^{-\left(x_{i}-\alpha\right) / \beta}\right]^{3}}$

Also we get $l_{30}=\frac{\partial^{3} L n L}{\partial \alpha^{3}}$ by the following expression:

$\frac{\partial^{3} L n L}{\partial \alpha^{3}}=\frac{-2}{\beta^{3}} \sum_{i=1}^{n} \frac{e^{-\left(x_{i}-\alpha\right) / \beta}-e^{-2\left(x_{i}-\alpha\right) / \beta}}{\left[1+e^{-\left(x_{i}-\alpha\right) / \beta}\right]^{3}}$

We obtain $l_{03}=\frac{\partial^{3} L n L}{\partial \beta^{3}}$ by the following expression:

$\frac{\partial L n L}{\partial \beta}=\sum_{i=1}^{n} \frac{\left(x_{i}-\alpha\right)}{\beta^{2}}-\frac{n}{\beta}-2 \sum_{i=1}^{n} \frac{\frac{\left(x_{i}-\alpha\right)}{\beta^{2}} e^{-\left(x_{i}-\alpha\right) / \beta}}{\left[1+e^{-\left(x_{i}-\alpha\right) / \beta}\right]}$

$\frac{\partial^{2} L n L}{\partial \beta^{2}}=-2 \sum_{i=1}^{n} \frac{x_{i}-\alpha}{\beta^{3}}+\frac{n}{\beta^{2}}-$

$2 \sum_{i=1}^{n} \frac{\frac{x_{i}-\alpha}{\beta^{3}} e^{-\left(x_{i}-\alpha\right) / \beta}\left[\frac{x_{i}-\alpha}{\beta} e^{-\left(x_{i}-\alpha\right) / \beta}-2-2 e^{-\left(x_{i}-\alpha\right) / \beta}\right]}{\left[1+e^{-\left(x_{i}-\alpha\right) / \beta}\right]^{2}}(21)$

$\frac{\partial^{3} L n L}{\partial \beta^{3}}=6 \sum_{i=1}^{n} \frac{x_{i}-\alpha}{\beta^{4}}-\frac{2 n}{\beta^{3}}-2 \sum_{i=1}^{n} \frac{\frac{\left(x_{i}-\alpha\right)^{3}}{\beta^{6}} e^{-\left(x_{i}-\alpha\right) / \beta}\left[1-e^{-\left(x_{i}-\alpha\right) / \beta}\right]}{\left[1+e^{-\left(x_{i}-\alpha\right) / \beta}\right]^{2}}-\frac{6\left(x_{i}-\alpha\right)^{2}}{\beta^{5}} e^{-\left(x_{i}-\alpha\right) / \beta}[1+$ $\left.e^{-\left(x_{i}-\alpha\right) / \beta}\right]+\frac{6\left(x_{i}-\alpha\right)^{2}}{\beta^{4}} e^{-\left(x_{i}-\alpha\right) / \beta}\left[1+2 e^{-2\left(x_{i}-\alpha\right) / \beta}+e^{-\left(x_{i}-\alpha\right) / \beta}\right](22)$

So when $R(\alpha, \beta)=\alpha$ then

$$
\begin{aligned}
& W_{1}=\frac{\partial R}{\partial \alpha}=1 \quad ; \quad W_{2}=\frac{\partial R}{\partial \beta}=0(23) \\
& W_{12}=W_{21}=\frac{\partial^{2} R}{\partial \alpha \partial \beta}=0(24) \\
& P=\ln f(\alpha, \beta)=\ln \left[c e^{-C \alpha} \frac{a^{n}}{\Gamma(n)} \beta^{n-1} e^{-\beta a}\right] \\
& P=\ln c-c \alpha+n \ln a-\ln \Gamma(n)+(n-1) \ln \beta-\beta a \\
& P 1=\frac{\partial P}{\partial \alpha}=-C(26) \quad P 2=\frac{\partial P}{\partial \beta}=\frac{n-1}{\beta}-a \\
& A=W_{12} T_{12}+W_{21} T_{21}=0 \\
& B_{12}=\left(W_{1} T_{12}+W_{2} T_{12}\right) T_{11}=W_{1} T_{12} T_{11} \\
& C_{12}=3 W_{1} T_{11} T_{12}+W_{1}\left(T_{11} T_{22}+2 T^{2}{ }_{12}\right) \\
& =3 T_{11} T_{12}+T_{11} T_{22}+2 T_{12}^{2} \\
& A_{12}=T_{12} A_{21}=T_{21}
\end{aligned}
$$

To compensate the value of $T_{11}, T_{22}, T_{12}, T_{22}$ by the equation Such that :$T_{11}=\frac{u}{D u-v^{2}} ; T_{22}=\frac{D}{D u-v^{2}} \quad ; \quad T_{12}=T_{21}=\frac{-v}{D u-v^{2}}(29)$ 
Where

$U=\frac{\partial^{2} \ln L}{\partial \beta^{2}}, D=\frac{\partial^{2} \ln L}{\partial \alpha^{2}}, V=\frac{\partial^{2} \ln L}{\partial \beta \partial \alpha}$

These derivative (U ,D) we calculated them in equations (21), (17).

And since $\frac{\partial^{2} \ln L}{\partial \alpha \partial \beta}=\frac{\partial^{2} \ln L}{\partial \beta \partial \alpha}$ then $(\mathrm{V})$ is the same equation (15)

Then the estimator $\alpha$ can be written as:-

$\hat{\alpha}=\hat{\alpha}_{m L E}+\frac{1}{2}\left[L_{30} T_{12} T_{11}+L_{03} T_{21} T_{22}+L_{21}\left(3 T_{11} T_{12}+T_{11} T_{22}+2 T^{2}{ }_{12}\right)\right]+$ $(-c) T_{12}+\quad\left(\frac{n-1}{\beta}-\alpha\right) T_{12}(30)$

Where $\mathrm{c}, \mathrm{a}$ are assumed to be any numbers $\mathrm{c}>0, \mathrm{a}>0$.

And finely when $R(\alpha, \beta)=\beta$

Then $W_{1}=0 ; W_{2}=1 ; \quad W_{12}=W_{21}=0 ; \quad \mathrm{A}=0$

$B_{12}=T_{12} T_{11} ; \quad B_{21}=T_{21} T_{22} ; \quad C_{12}=0 \quad ; C_{21}=3 T_{22} T_{21}+T_{22} T_{11}+$ $2 T^{2}{ }_{21}$

$A_{21}=A_{12}=T_{21}$

Then the estimator $\beta$ writer as follows

$\hat{\beta}=\hat{\beta}_{m L E}+\frac{1}{2}\left[L_{30} T_{12} T_{11}+L_{03} T_{21} T_{22}+L_{21}\left(3 T_{22} T_{21}+T_{11} T_{22}+2 T^{2}{ }_{21}\right)\right]+$

$(-c) T_{21}+\quad\left(\frac{n-1}{\beta}\right) T_{21}$

\section{Rank Sampling Set estimator} Method(RSSE):-

One key of statistical inference is estimating the parameters of the distribution from the exist samples .one of the most common mechanisms for obtaining such data is that of a simple random sample. The concept of rank set sampling is arecent development that enable one to provide more structure to the collected sample items, this approach to data collection was prevailed for situations where taking the actual measurement for sample observations is difficult (costly ,destructive, time- consuming), for this reason rank set sampling is used widely in (eniromental, ecological ,agricultural, medical studies).

This method first proposed by Mcintyre(1952) the mean of pasture yields he was interested in improving the precision of arable crops without a substantial increase in the number of fields from which detailed expensive and tedious measurement need to be collected[11] .

Halls-Dell(1966)who established that rank set sampling was more efficient than simple random sampling estimating the population mean[12].

Dell and Clutler (1972) demonstrated that rank set sampling is more efficient than simple random sample even when the errors are ranking is present[12].

The procedure to find estimator for logistic distribution by Rank set sampling as follows:-

Let $\quad x_{1}, x_{2} \ldots \ldots x_{m}$ be a random sample from logistic distribution ; assumed that $x_{(1)}, x_{(2)} \ldots \ldots . x_{(m)}$ be the order statistics obtained by ordering the sample in increasing order .

The probability density function ( pdf ) of order statistic $x_{i}$ is:

$f\left(x_{i}\right)=\frac{n !}{(i-1) !(n-i) !}\left[F\left(x_{i}\right)\right]^{i-1}\left[1-F\left(x_{i}\right)\right]^{n-i} f\left(x_{i}\right)$

$f\left(x_{i}\right)=\frac{n !}{(i-) !(n-i) !}\left[\frac{1}{1+e^{-\left(x_{i}^{-\alpha}\right) / \beta}}\right]^{i-1}\left[\frac{e^{-\left(x_{i}^{-\alpha}\right) / \beta}}{1+e^{-\left(x_{i}-\alpha\right) / \beta}}\right]^{n-i} \cdot \frac{e^{-\left(x_{i}-\alpha\right) / \beta}}{\beta\left(1+e^{-\left(x_{i}-\alpha\right) / \beta}\right)^{2}}$

suppose that $K=\frac{n !}{(i-1) !(n-i) !} \operatorname{thenf}\left(x_{i}\right)=K\left[\frac{1}{1+e^{-\left(x_{i-\alpha}\right) / \beta}}\right]^{n+1} \beta^{-1}\left[e^{-\left(x_{i}-\alpha\right)}\right]^{n-i+1}$ 
The likelihood function of the order sample $x_{(1)}, x_{(2)} \ldots \ldots x_{(m)}$ is:-

$L\left(x_{(1)}, x_{(2)} \ldots \ldots . x_{(m)} ; \alpha, \beta\right)=K^{n} \beta^{-n} \prod_{i=1}^{n}\left[\frac{1}{1+e^{-\left(x_{i}-\alpha\right) / \beta}}\right]^{n+1} \cdot \prod_{i=1}^{n}\left[e^{-\left(x_{i}-\alpha\right) / \beta}\right]^{n-i+1}$

$L=K^{n} \beta^{-n} \prod_{i=1}^{n}\left[1+e^{-\left(x_{i}-\alpha\right) / \beta}\right]^{-n-1} \prod_{i=1}^{n}\left[e^{-\left(x_{i}-\alpha\right) / \beta}\right]^{n-i+1}$

The $\log -$ likelihood function is :-

$L n L=n \ln k-(n+1) \sum_{i=1}^{n} \ln \left[1+e^{-\left(x_{i}-\alpha\right) / \beta}\right]-n \ln \beta-\sum_{i=1}^{n}(n-i+1)\left(\frac{x_{i}-\alpha}{\beta}\right)(37)$

By finding the first derivative of (37) with respect to $\alpha, \beta$ and equating them to zero, as follows :-

$\frac{\partial \ln L}{\partial \alpha}=-(n+1) \sum_{i=1}^{n} \frac{e^{-\left(x_{i}-\alpha\right) / \beta} \cdot \frac{1}{\beta}}{\left[1+e^{-\left(x_{i}-\alpha\right) / \beta}\right]}+\frac{1}{\beta} \sum_{i=1}^{n}(n-i+1)$

$\frac{\partial \ln L}{\partial \alpha}=\frac{1}{\beta} \sum_{i=1}^{n}(n-i+1)-\frac{n+1}{\beta} \sum_{i=1}^{n} \frac{e^{-\left(x_{i}-\alpha\right) / \beta}}{1+e^{-\left(x_{i}-\alpha\right) / \beta}}$

$\frac{\partial \ln L}{\partial \beta}=-(n+1) \sum_{i=1}^{n} \frac{e^{-\left(x_{i}-\alpha\right) / \beta}\left(\frac{x_{i}-\alpha}{\beta^{2}}\right)}{1+e^{-\left(x_{i}-\alpha\right) / \beta}}-\frac{n}{\beta}+\sum_{i=1}^{n}(n-i+1)\left(\frac{x_{i}-\alpha}{\beta^{2}}\right)$

$\frac{\partial \ln L}{\partial \beta}=\sum_{i=1}^{n} \frac{(n-i+1)\left(X_{i}-\alpha\right)}{\beta^{2}}-\frac{n}{\beta}-\frac{(n+1)}{\beta^{2}} \sum_{i=1}^{n} \frac{\left(X_{i}-\alpha\right) e^{-\left(x_{i}-\alpha\right) / \beta}}{1+e^{-\left(x_{i}-\alpha\right) / \beta}}(39)$

To solve these non-linear equations we use the iterative method which is Newton Raphson method and it's steps as follows:-

$\left[\begin{array}{c}\alpha_{i+1} \\ \beta_{i+1}\end{array}\right]=\left[\begin{array}{l}\alpha_{i} \\ \beta_{i}\end{array}\right]+J^{-1}\left[\begin{array}{l}f\left(\alpha_{i}\right) \\ f\left(\beta_{i}\right)\end{array}\right]$

To find the jacobian which is :-

$|J|=\left[\begin{array}{l}\frac{\partial f(\alpha)}{\partial \alpha} \frac{\partial f(\alpha)}{\partial \beta} \\ \frac{\partial f(\beta)}{\partial \alpha} \frac{\partial f(\beta)}{\partial \beta}\end{array}\right]$

First we assuming that the derivate of $\ln \mathrm{L}$ with respect to $\alpha$ and $\beta$ as function such that :

$\frac{\partial \ln L}{\partial \alpha}=f(\alpha) \frac{\partial \ln L}{\partial \beta}=f(\beta)$

Then the first drivite to $\mathrm{f}(\alpha)$ and $\mathrm{f}(\beta)$ with respect to $\alpha$ and $\beta$ as follows:-

$$
\begin{aligned}
& \frac{\partial f(\alpha)}{\partial \alpha}=\frac{-(n+1)}{\beta} \sum_{i=1}^{n} \frac{\left[1+e^{-\left(x_{i}-\alpha\right) / \beta}\right] e^{-\left(x_{i}-\alpha\right) / \beta} \cdot \frac{1}{\beta}-e^{-\left(x_{i}-\alpha\right) / \beta} e^{-\left(x_{i}-\alpha\right) / \beta} \cdot \frac{1}{\beta}}{\left[1+e^{-\left(x_{i}-\alpha\right) / \beta}\right]^{2}} \\
& \frac{\partial f(\alpha)}{\partial \alpha}=\frac{-(n+1)}{\beta^{2}} \sum_{i=1}^{n} \frac{e^{-\left(x_{i}^{-\alpha}\right) / \beta}}{\left[1+e^{-\left(x_{i}^{-\alpha}\right) / \beta}\right]^{2}} \\
& \frac{\partial f(\alpha)}{\partial \beta}=\frac{-1}{\beta^{2}} \sum_{i=1}^{n}(n-i+1)-(n+1) \sum_{i=1}^{n} \frac{\left[\beta+\beta e^{-\left(x_{i}-\alpha\right) / \beta}\right] e^{-\left(x_{i}-\alpha\right) / \beta}\left(\frac{x_{i}-\alpha}{\beta^{2}}\right)}{\left[\beta+\beta e^{-\left(x_{i}-\alpha\right) / \beta}\right]^{2}} \\
& -e^{-\left(x_{i}-\alpha\right) / \beta}\left[1+\beta e^{-\left(x_{i}-\alpha\right) / \beta} \cdot \frac{x_{i}-\alpha}{\beta^{2}}+e^{-\left(x_{i}-\alpha\right) / \beta}\right] \\
& \frac{\partial f(\alpha)}{\partial \beta}=\frac{-1}{\beta^{2}} \sum_{i=1}^{n}(n-i+1)-\frac{n+1}{\beta^{2}} \sum_{i=1}^{n} \frac{e^{-\left(x_{i}-\alpha\right) / \beta}\left[\frac{x_{i}-\alpha}{\beta}-1-e^{-\left(x_{i}-\alpha\right) / \beta}\right]}{\left[1+e^{-\left(x_{i}^{-\alpha}\right) / \beta}\right]^{2}}
\end{aligned}
$$




$$
\begin{aligned}
& \frac{\partial f(\beta)}{\partial \alpha}=\sum_{i=1}^{n} \frac{(n-i+1)}{\beta^{2}}(-1) \\
& -\frac{(n+1)}{\beta^{2}} \sum_{i=1}^{n} \frac{\left[1+e^{-\left(x_{i}-\alpha\right) / \beta}\right]\left[e^{-\left(x_{i}-\alpha\right) / \beta}(-1)+\left(x_{i}-\alpha\right) e^{-\left(x_{i}-\alpha\right) / \beta} \frac{1}{\beta}\right]}{\left[1+e^{-\left(x_{i}-\alpha\right) / \beta}\right]^{2}} \\
& -\left[\left(\mathrm{x}_{\mathrm{i}}-\alpha\right) \mathrm{e}^{-\left(\mathrm{x}_{\mathrm{i}}-\alpha\right) / \beta} \mathrm{e}^{-\left(\mathrm{x}_{\mathrm{i}}-\alpha\right) / \beta} \cdot \frac{1}{\beta}\right] \\
& \frac{\partial f(\beta)}{\partial \alpha}=\sum_{i=1}^{n} \frac{-(n+1)}{\beta^{2}}-\frac{(n+1)}{\beta^{2}} \sum_{i=1}^{n} \frac{e^{-\left(x_{i}-\alpha\right) / \beta}\left[\frac{x_{i}-\alpha}{\beta}-1-e^{-\left(x_{i}-\alpha\right) / \beta}\right]}{\left[1+e^{-\left(x_{i}-\alpha\right) / \beta}\right]^{2}}(44) \\
& \frac{\partial f(\beta)}{\partial \beta}= \\
& -2 \sum_{i=1}^{n} \frac{\left(x_{i}-\alpha\right)(n-i+1)}{\beta^{3}} \frac{n}{\beta^{2}}-(\mathrm{n}+1) \sum_{i=1}^{n} \frac{\left(1+e^{-\left(x_{i}^{-\alpha}\right) / \beta}\right)\left[e^{-\left(x_{i}^{-\alpha}\right) / \beta}-(-2) \frac{x_{i}-\alpha}{\beta^{3}}+\frac{x_{i}-\alpha}{\beta^{2}} e^{-\left(x_{i}-\alpha\right) / \beta} \frac{x_{i}-\alpha}{\beta^{2}}\right]}{\left[1+e^{-\left(x_{i}-\alpha\right) / \beta}\right]^{2}} \\
& -\frac{\left(\frac{x_{i}-\alpha}{\beta^{2}} e^{-\left(x_{i}-\alpha\right) / \beta}\right) e^{-\left(x_{i}-\alpha\right) / \beta} \frac{x_{i}-\alpha}{\beta^{2}}}{\left[1+e^{-(\mathrm{xi}-\alpha) / \beta}\right]^{2}} \\
& \frac{\partial f(\beta)}{\partial \beta}= \\
& -2 \sum_{i=1}^{n} \frac{(\mathrm{xi}-\alpha)(n-i+1)}{\beta^{3}}+\frac{n}{\beta^{2}}-(n+1) \sum_{i=1}^{n} \frac{e^{-\left(x_{i}-\alpha\right) / \beta}\left[-2\left(\frac{x_{i}-\alpha}{\beta^{3}}\right)-2\left(\frac{x_{i}-\alpha}{\beta^{3}}\right) e^{-\left(x_{i}-\alpha\right) / \beta}+\left(\frac{x_{i}-\alpha}{\beta^{2}}\right)^{2}\right]}{\left[1+e^{-\left(x_{i}^{-\alpha}\right) / \beta}\right]^{2}}
\end{aligned}
$$

And to terminated Newton Raphson method we use the equation :-

$\in=\llbracket\left[\begin{array}{c}\alpha_{i+1} \\ \beta_{i+1}\end{array}\right]-\left[\begin{array}{c}\alpha_{i} \\ \beta_{i}\end{array}\right] \mid$ where $\in$ is assumed value

\section{Numerical results and Comments :}

In this section; simulation technique used to generate many various of samples by using Monte Carlo method, to compare between the methods of estimation which are mentioned in previous section .

First : generating random numbers which distributed as uniform distribution with $[0,1]$; then transform these random numbers to random variable which distributed as logistic distribution by using inverse transformation method which depend on cumulative distribution function for logistic distribution as follows:

$$
\begin{aligned}
& F\left(x_{i}\right)=u=\frac{1}{1+e^{-(x-\alpha) / \beta}} \quad ; \quad \mathrm{X}=\alpha- \\
& \beta \ln \left(\frac{1}{u}-1\right) \ldots . .(47)
\end{aligned}
$$

Second: To generate $\mathrm{x}$ which distributed as logistic function, we must choose many value to the parameter $\alpha$ and $\beta$ as well as we must choose many various samples sizes which are as follows:

$\alpha: 0.25,0.75,1.5,2.5$; ,2.5; $\mathrm{n}: 10,20,50$

$\beta: 0.5,1$

And we replication the data of experiment (1000) times, then the number of all generating experiment is(12) times.

Third: By utilizing program visual basic ,we have got the following estimated values for the location and scale parameter to the logistic distribution and numerical results scheduleted in table1 
Fourth: Computing the Mean squares error measure (MSE)and Mean absolute percentage error
measure(MAPE) for all situations studied in this paper and scheduleted in the tables (2) and (3).

Table (1) the estimate values for parameters $\alpha$ and $\beta$ When $\mathrm{n}=10$

\begin{tabular}{|c|c|c|c|c|c|}
\hline \multirow{2}{*}{$\boldsymbol{\alpha}$} & \multirow{2}{*}{$\boldsymbol{\beta}$} & \multicolumn{2}{|l|}{ RSSE } & \multicolumn{2}{|l|}{ LAE } \\
\hline & & $\widehat{\alpha}$ & $\widehat{\boldsymbol{\beta}}$ & $\widehat{\alpha}$ & $\widehat{\boldsymbol{\beta}}$ \\
\hline \multirow[t]{3}{*}{0.25} & 0.5 & 0.172784 & $\mathbf{0 . 4 1 5 8 3 7}$ & 0.276769 & 0.717009 \\
\hline & 1 & 0.128955 & 0.708412 & 0.057022 & 0.853903 \\
\hline & 2.5 & 0.185008 & 2.287435 & 0.777169 & 2.62177 \\
\hline \multirow[t]{3}{*}{0.75} & 0.5 & 0.72542 & 0.331941 & 0.877954 & 0.511009 \\
\hline & 1 & 0.660448 & 0.820309 & $\mathbf{0 . 5 5 3 1 8 1}$ & 0.969319 \\
\hline & 2.5 & 0.896211 & 2.019816 & 0.764394 & 2.212687 \\
\hline \multirow[t]{3}{*}{1.5} & 0.5 & 1.620975 & 0.40149 & 1.472668 & 0.500236 \\
\hline & 1 & 1.302785 & 0.840384 & 1.590418 & 1.321985 \\
\hline & 2.5 & 1.266374 & 2.346401 & $\mathbf{1 . 5 5 3 7 7 5}$ & 2.57828 \\
\hline \multirow[t]{3}{*}{2.5} & 0.5 & 2.40465 & 0.366753 & 2.572956 & 0.520779 \\
\hline & 1 & 2.595824 & 0.958468 & 2.434724 & 1.227105 \\
\hline & 2.5 & 2.281461 & 2.310971 & 3.167246 & 2.636337 \\
\hline
\end{tabular}

When $\mathrm{n}=20$

\begin{tabular}{|c|c|c|c|c|c|}
\hline \multirow{2}{*}{$\alpha$} & \multirow{2}{*}{$\boldsymbol{\beta}$} & \multicolumn{2}{|l|}{ RSSE } & \multicolumn{2}{|l|}{ LAE } \\
\hline & & $\widehat{\alpha}$ & $\widehat{\boldsymbol{\beta}}$ & $\widehat{\alpha}$ & $\widehat{\boldsymbol{\beta}}$ \\
\hline \multirow[t]{3}{*}{0.25} & 0.5 & 0.280343 & 0.435591 & 0.253419 & 0.454164 \\
\hline & 1 & 0.253055 & 0.878647 & 0.417546 & $\mathbf{0 . 9 9 7 8 9 7}$ \\
\hline & 2.5 & 0.629894 & 2.523152 & 0.39713 & 1.948425 \\
\hline \multirow[t]{3}{*}{0.75} & 0.5 & 0.802783 & 0.562325 & 0.787021 & 0.415198 \\
\hline & 1 & 0.946529 & 0.782279 & 0.774733 & 0.908841 \\
\hline & 2.5 & 1.143741 & 2.071451 & 0.387115 & 2.615597 \\
\hline \multirow[t]{3}{*}{1.5} & 0.5 & 1.503476 & 0.537935 & 1.427472 & 0.575071 \\
\hline & 1 & 1.406217 & 1.021662 & 1.853357 & 0.80789 \\
\hline & 2.5 & 1.273903 & 2.309122 & 2.088239 & 2.97301 \\
\hline \multirow[t]{3}{*}{2.5} & 0.5 & 2.53223 & 0.499329 & 2.568995 & 0.476806 \\
\hline & 1 & 2.63562 & 1.116876 & 2.650861 & 1.07464 \\
\hline & 2.5 & 2.529316 & 2.101214 & 2.379594 & 2.623302 \\
\hline
\end{tabular}

When $\mathrm{n}=50$

\begin{tabular}{|c|c|c|c|c|c|}
\hline \multirow{2}{*}{$\alpha$} & \multirow{2}{*}{$\boldsymbol{\beta}$} & \multicolumn{2}{|c}{ RSSE } & \multicolumn{2}{c|}{ LAE } \\
\cline { 3 - 6 } & & $\widehat{\boldsymbol{\alpha}}$ & $\widehat{\boldsymbol{\beta}}$ & $\widehat{\boldsymbol{\alpha}}$ & $\boldsymbol{\beta}$ \\
\hline 0.25 & 0.5 & 0.249615 & 0.474637 & 0.260385 & 0.397702 \\
\hline & 1 & 0.178343 & 0.973637 & 0.088408 & 1.002549 \\
\hline & 2.5 & 0.1199 & 2.614521 & 0.322254 & 2.503849 \\
\hline 0.75 & 0.5 & 0.795641 & 0.564298 & 0.740074 & 0.456381 \\
\hline & 1 & 0.757543 & 1.025116 & 0.784252 & 0.872466 \\
\hline & 2.5 & 0.923732 & 2.339286 & 0.655519 & 2.177922 \\
\hline 1.5 & 0.5 & 1.503773 & 0.507133 & 1.509202 & 0.460897 \\
\hline & 1 & 1.554546 & 1.104526 & 1.395621 & 0.986348 \\
\hline & 2.5 & 1.536871 & 2.697889 & 1.505954 & 2.557208 \\
\hline 2.5 & 0.5 & 2.496831 & 0.520751 & 2.497792 & 0.466096 \\
\hline & 1 & 2.425772 & 0.773515 & 2.57641 & 1.020535 \\
\hline & 2.5 & 2.583397 & 2.583399 & 2.463726 & 2.862835 \\
\hline & & & & & \\
\hline
\end{tabular}

From table (1) we can make the following comments:

1-Noting that estimated values of parameter $\alpha$ are vibrating for all samples size in Rank sampling set method; while the estimate values of $\alpha$ are vibrating for all samples size in lindley approximation Bayes method. 2-Showing that the estimated values of parameter $\alpha$ are converge to the true values of $\alpha$ (19) times in Rank sampling set method; but the estimated values of $\alpha$ converge to true values of $\alpha$ (17) times in lindley approximation Bayes method.

3-Observing that the estimated values of parameter $\beta$ are vibrating for all samples sizes of two studied methods. 4-Showing that the estimated values of $\beta$ are converge to the true value of $\beta$ 
(20) times for lindley approximation

Bayes method and converge to the true values of $\beta$ (16) times by using Rank sampling set method .
Table (2)MSE for $\widehat{\alpha}$ and $\widehat{\boldsymbol{\beta}}$

When $\mathrm{n}=10$
When $\mathrm{n}=20$

\begin{tabular}{|c|c|c|c|c|c|}
\hline \multirow{2}{*}{$\alpha$} & \multirow{2}{*}{$\beta$} & \multicolumn{2}{|c|}{$\operatorname{MSE}(\widehat{\boldsymbol{\alpha}})$} & \multicolumn{2}{|c|}{$\operatorname{MSE}(\hat{\boldsymbol{\beta}})$} \\
\cline { 3 - 6 } & & RSSE & LAE & RSSE & LAE \\
\hline $\mathbf{0 . 2 5}$ & $\mathbf{0 . 5}$ & $\mathbf{0 . 0 1 7 4 4 6}$ & $\mathbf{0 . 0 6 7 6 2 2}$ & $\mathbf{0 . 0 4 1 9 4 3}$ & $\mathbf{0 . 0 6 4 9 5 6}$ \\
\hline & 1 & $\mathbf{0 . 2 0 1 1 6 6}$ & $\mathbf{0 . 0 5 2 4 5 2}$ & $\mathbf{0 . 1 1 4 6 2 9}$ & $\mathbf{0 . 0 2 6 4 4 9}$ \\
\hline & $\mathbf{2 . 5}$ & $\mathbf{0 . 3 7 1 2 3 7}$ & $\mathbf{1 . 3 5 6 3 3 4}$ & $\mathbf{0 . 1 6 7 6 5}$ & $\mathbf{1 . 1 5 5 1 9}$ \\
\hline $\mathbf{0 . 7 5}$ & $\mathbf{0 . 5}$ & $\mathbf{0 . 0 0 6 1 0 3}$ & $\mathbf{0 . 1 0 7 0 5 9}$ & $\mathbf{0 . 0 3 9 1 1 8}$ & $\mathbf{0 . 0 5 4 8 7 8}$ \\
\hline & 1 & $\mathbf{0 . 0 4 6 6}$ & $\mathbf{0 . 0 4 5 9 5 3}$ & $\mathbf{0 . 0 3 9 6 5 1}$ & $\mathbf{0 . 1 0 1 4 4 1}$ \\
\hline & $\mathbf{2 . 5}$ & $\mathbf{0 . 3 7 0 1 7 2}$ & $\mathbf{0 . 1 0 1 9 2 8}$ & $\mathbf{0 . 7 5 6 2 7}$ & $\mathbf{0 . 2 6 5 1 7}$ \\
\hline 1.5 & $\mathbf{0 . 5}$ & $\mathbf{0 . 0 1 7 9 0 9}$ & $\mathbf{0 . 0 1 0 3 1 7}$ & $\mathbf{0 . 0 3 7 1 0 9}$ & $\mathbf{0 . 0 1 0 3 2 6}$ \\
\hline & 1 & $\mathbf{0 . 0 6 0 1 4 4}$ & $\mathbf{0 . 0 2 8 5 6 4}$ & $\mathbf{0 . 0 4 7 7 0 4}$ & $\mathbf{0 . 1 7 7 4 3 3}$ \\
\hline & $\mathbf{2 . 5}$ & $\mathbf{0 . 2 7 7 1 9 8}$ & $\mathbf{0 . 2 7 2 5 8}$ & $\mathbf{0 . 0 5 4 9 1 2}$ & $\mathbf{0 . 3 6 5 7 6 6}$ \\
\hline $\mathbf{2 . 5}$ & $\mathbf{0 . 5}$ & $\mathbf{0 . 0 1 4 5 3 4}$ & $\mathbf{0 . 0 2 8 0 6 3}$ & $\mathbf{0 . 0 4 9 0 5 2}$ & $\mathbf{0 . 0 0 7 3 5 8}$ \\
\hline & 1 & $\mathbf{0 . 0 5 5 8 7 7}$ & $\mathbf{0 . 1 0 0 6 9 4}$ & $\mathbf{0 . 0 3 5 0 7 4}$ & $\mathbf{0 . 0 7 7 6 6 2}$ \\
\hline & $\mathbf{2 . 5}$ & $\mathbf{0 . 6 1 7 9 9 3}$ & $\mathbf{2 . 3 7 4 3 4 2}$ & $\mathbf{0 . 1 2 3 1 5 6}$ & $\mathbf{0 . 1 2 7 7 7 4}$ \\
\hline & & & & & \\
\hline
\end{tabular}

\begin{tabular}{|c|c|c|c|c|c|}
\hline \multirow{2}{*}{$\alpha$} & \multirow{2}{*}{$\beta$} & \multicolumn{2}{|c|}{$\operatorname{MSE}(\widehat{\boldsymbol{\alpha}})$} & \multicolumn{2}{c|}{$\operatorname{MSE}(\hat{\beta})$} \\
\cline { 3 - 6 } & & RSSE & LAE & RSSE & LAE \\
\hline $\mathbf{0 . 2 5}$ & $\mathbf{0 . 5}$ & $\mathbf{0 . 0 1 5 8 9 5}$ & $\mathbf{0 . 0 0 3 9 5 4}$ & $\mathbf{0 . 0 3 1 1 6 5}$ & $\mathbf{0 . 0 0 2 5 4 4}$ \\
\hline & 1 & $\mathbf{0 . 0 2 2 0 9 8}$ & $\mathbf{0 . 0 6 1 8 4}$ & $\mathbf{0 . 0 4 9 5 2 1}$ & $\mathbf{0 . 0 1 0 7 9 8}$ \\
\hline & $\mathbf{2 . 5}$ & $\mathbf{0 . 3 6 5 3 6 9}$ & $\mathbf{0 . 1 0 6 7 2 2}$ & $\mathbf{0 . 1 3 4 2 0 4}$ & $\mathbf{0 . 3 9 5 2 9 8}$ \\
\hline $\mathbf{0 . 7 5}$ & $\mathbf{0 . 5}$ & $\mathbf{0 . 0 0 7 6 3 9}$ & $\mathbf{0 . 0 0 6 6 6 8}$ & $\mathbf{0 . 0 1 9 2 0 2}$ & $\mathbf{0 . 0 1 8 1 2}$ \\
\hline & 1 & $\mathbf{0 . 0 8 8 6 2 6}$ & $\mathbf{0 . 0 2 5 4 6 9}$ & $\mathbf{0 . 0 6 4 4 5 3}$ & $\mathbf{0 . 0 4 2 2 4 2}$ \\
\hline & $\mathbf{2 . 5}$ & $\mathbf{0 . 4 4 1 0 2 1}$ & $\mathbf{0 . 2 3 4 5 3 1}$ & $\mathbf{0 . 1 8 6 9 7 3}$ & $\mathbf{0 . 1 9 2 2 2 8}$ \\
\hline $\mathbf{1 . 5}$ & $\mathbf{0 . 5}$ & $\mathbf{0 . 0 1 9 4 7 9}$ & $\mathbf{0 . 0 0 7 4 9 9}$ & $\mathbf{0 . 0 3 9 1 7 2}$ & $\mathbf{0 . 0 1 3 9 3 3}$ \\
\hline & 1 & $\mathbf{0 . 0 4 9 7 0 1}$ & $\mathbf{0 . 1 8 2 5 6}$ & $\mathbf{0 . 0 5 5 1 9 4}$ & $\mathbf{0 . 0 4 0 1 4 5}$ \\
\hline & $\mathbf{2 . 5}$ & $\mathbf{0 . 2 0 0 9 1 4}$ & $\mathbf{0 . 6 4 4 7 2 9}$ & $\mathbf{0 . 2 3 7 5 3 8}$ & $\mathbf{0 . 2 9 1 2 4 6}$ \\
\hline $\mathbf{2 . 5}$ & $\mathbf{0 . 5}$ & $\mathbf{0 . 0 1 7 4 4 8}$ & $\mathbf{0 . 0 1 8 9 0 8}$ & $\mathbf{0 . 0 4 8 6 3 5}$ & $\mathbf{0 . 0 0 2 7 3 4}$ \\
\hline & $\mathbf{1}$ & $\mathbf{0 . 0 7 8 5 2 7}$ & $\mathbf{0 . 0 3 0 4 0 4}$ & $\mathbf{0 . 0 1 4 1 7}$ & $\mathbf{0 . 0 2 0 7 5}$ \\
\hline & $\mathbf{2 . 5}$ & $\mathbf{0 . 0 3 3 7 1 8}$ & $\mathbf{0 . 1 0 6 7 9 1}$ & $\mathbf{0 . 2 2 3 7 4 7}$ & $\mathbf{0 . 1 8 6 0 1 7}$ \\
\hline & & & & & \\
\hline
\end{tabular}

When $\mathrm{n}=50$

\begin{tabular}{|l|l|l|l|l|l|}
\hline \multirow{2}{*}{$\alpha$} & \multirow{2}{*}{$\beta$} & \multicolumn{2}{|l|}{$\operatorname{MSE}(\widehat{\boldsymbol{\alpha}})$} & \multicolumn{2}{l|}{$\operatorname{MSE}(\hat{\beta})$} \\
\cline { 3 - 6 } & & RSSE & LAE & RSSE & LAE \\
\hline $\mathbf{0 . 2 5}$ & $\mathbf{0 . 5}$ & $\mathbf{0 . 0 0 1 3 1 5}$ & $\mathbf{0 . 0 0 2 7 1 1}$ & $\mathbf{0 . 0 0 1 3 4 1}$ & $\mathbf{0 . 0 1 4 7 3 1}$ \\
\hline & $\mathbf{1}$ & $\mathbf{0 . 0 1 5 2 1 9}$ & $\mathbf{0 . 0 4 9 5 3 3}$ & $\mathbf{0 . 0 1 5 0 9 7}$ & $\mathbf{0 . 0 0 6 7 7 4}$ \\
\hline & $\mathbf{2 . 5}$ & $\mathbf{0 . 0 8 6 5 3 2}$ & $\mathbf{0 . 4 4 8 3 3 3}$ & $\mathbf{0 . 0 2 4 0 3 6}$ & $\mathbf{0 . 0 6 4 3 8 1}$ \\
\hline $\mathbf{0 . 7 5}$ & $\mathbf{0 . 5}$ & $\mathbf{0 . 0 0 8 0 7 8}$ & $\mathbf{0 . 0 0 0 5 0 6}$ & $\mathbf{0 . 0 1 9 7 9 1}$ & $\mathbf{0 . 0 0 2 9 5 7}$ \\
\hline & $\mathbf{1}$ & $\mathbf{0 . 0 0 7 0 8 7}$ & $\mathbf{0 . 0 2 2 4 0 3}$ & $\mathbf{0 . 0 1 8 3 2 3}$ & $\mathbf{0 . 0 2 0 2 5 8}$ \\
\hline & $\mathbf{2 . 5}$ & $\mathbf{0 . 0 8 4 3 5}$ & $\mathbf{0 . 0 4 6 2 9 6}$ & $\mathbf{0 . 2 3 3 8 3 2}$ & $\mathbf{0 . 1 3 4 7 9 6}$ \\
\hline $\mathbf{1 . 5}$ & $\mathbf{0 . 5}$ & $\mathbf{0 . 0 0 8 9 9 8}$ & $\mathbf{0 . 0 0 4 3 7 4}$ & $\mathbf{0 . 0 1 7 2 3}$ & $\mathbf{0 . 0 0 4 6 0 8}$ \\
\hline & $\mathbf{1}$ & $\mathbf{0 . 0 2 6 8 6 3}$ & $\mathbf{0 . 0 2 7 3 3}$ & $\mathbf{0 . 0 7 0 3 2 6}$ & $\mathbf{0 . 0 0 2 7 0 3}$ \\
\hline & $\mathbf{2 . 5}$ & $\mathbf{0 . 0 2 7 6 2 5}$ & $\mathbf{0 . 0 6 6 0 3 8}$ & $\mathbf{0 . 0 4 9 9 8 6}$ & $\mathbf{0 . 0 1 0 5 3}$ \\
\hline $\mathbf{2 . 5}$ & $\mathbf{0 . 5}$ & $\mathbf{0 . 0 1 1 7 2 6}$ & $\mathbf{0 . 0 0 3 0 6 2}$ & $\mathbf{0 . 0 1 6 3 6 1}$ & $\mathbf{0 . 0 0 1 5 1 2}$ \\
\hline & $\mathbf{1}$ & $\mathbf{0 . 0 1 2 4 8 1}$ & $\mathbf{0 . 0 3 0 4 1 1}$ & $\mathbf{0 . 0 5 4 9 4 9}$ & $\mathbf{0 . 0 0 2 6 6 8}$ \\
\hline & $\mathbf{2 . 5}$ & $\mathbf{0 . 1 4 9 6 7}$ & $\mathbf{0 . 0 1 8 8 6 2}$ & $\mathbf{0 . 0 1 9 4 2 5}$ & $\mathbf{0 . 1 4 5 0 1 7}$ \\
\hline
\end{tabular}

From table(2) we can make the following comments:

1-The values of Mean squares error for $\widehat{\boldsymbol{\alpha}}$ are increasing where the samples sizes are increasing for all values of $\alpha, \beta$ and $n$ except when $\alpha=2.5, n=20$ which are vibrating for Rank sampling set method while the values of MSE for $\widehat{\boldsymbol{\alpha}}$ are increasing for all values of $\alpha, \beta$ and $n$ except when $\alpha=0.75$ and $\mathrm{n}=10$ are vibrating for lindley approximation Bayes method.

2-Noting that the values of MSE are vibrating for all increasing value of $\alpha$ and $\beta$.The smallest values of MSE are $(0.006103)$ when $\quad(\alpha=0.75 \quad, \beta=0.5$
$, \mathrm{n}=10)$ for Rank sampling set method $;(0.003954)$ when $\quad(\alpha=0.25, \quad \beta=0.5$ $, \mathrm{n}=20)$ for Lindley approximation method, and(0.000506)when $(\alpha=0.75$, $\beta=0.5, n=50)$ for Rank sampling set method.

3-Showing that the values of MSE for $\hat{\beta}$ are vibrating for all increasing values of $\alpha$ and $\beta$. The smallest values of MSE is (0.007358) for $(\alpha=2.5, \beta=0.5, n=10) \quad$ for Lindley approximation method, while MSE is $(0.002544)$ for $(\alpha=0.25, \beta=0.5, n=20)$ for Lindley approximation method, and MSE is $(0.001341)$ for 
$(\alpha=0.25, \beta=0.5, n=50) \quad$ for $\quad$ Rank sampling set method.

another time with respect to the 4-The values of MSE for $\hat{\beta}$ are increasing one time and vibrating increasing of value $\alpha$ and $\beta$.

5-The values of MSE $\hat{\beta}$ are vibrating for all values of increasing samples size.

Table (3) MAPE for $\widehat{\alpha}$ and $\hat{\beta}$

When $\mathrm{n}=10$

\begin{tabular}{|l|l|l|l|l|l|}
\hline \multirow{2}{*}{$\alpha$} & \multirow{3}{*}{$\beta$} & \multicolumn{2}{|l|}{ MAPE $(\widehat{\boldsymbol{\alpha}})$} & \multicolumn{2}{l|}{ MAPE $\widehat{\boldsymbol{\beta}})$} \\
\cline { 3 - 6 } & & RSSE & LAE & RSSE & LAE \\
\hline 0.25 & 0.5 & 0.8374 & 0.877625 & 0.298972 & 0.434018 \\
\hline & 1 & 0.817473 & 0.771914 & 0.322175 & 0.146274 \\
\hline & 2.5 & 4.011257 & 3.9194 & 0.18631 & 0.414818 \\
\hline 0.75 & 0.5 & 0.482715 & 0.407009 & 0.626506 & 0.423198 \\
\hline & 1 & 0.272401 & 0.262435 & 0.304109 & 0.295563 \\
\hline & 2.5 & 0.462407 & 0.35429 & 0.217135 & 0.198884 \\
\hline 1.5 & 0.5 & 0.069453 & 0.0659 & 0.214467 & 0.191021 \\
\hline & 1 & 0.107675 & 0.087696 & 0.250152 & 0.321833 \\
\hline & 2.5 & 0.324603 & 0.315722 & 0.054297 & 0.229494 \\
\hline 2.5 & 0.5 & 0.051248 & 0.046884 & 0.20317 & 0.142623 \\
\hline & 1 & 0.119575 & 0.119323 & 0.04002 & 0.227105 \\
\hline & 2.5 & 0.46519 & 0.466957 & 0.218477 & 0.141795 \\
\hline
\end{tabular}

When $n=20$

\begin{tabular}{|l|l|l|l|l|l|}
\hline \multirow{2}{*}{$\alpha$} & \multirow{3}{*}{$\boldsymbol{\beta}$} & \multicolumn{2}{|l|}{ MAPE $(\widehat{\boldsymbol{\alpha}})$} & \multicolumn{2}{l|}{ MAPE $(\widehat{\boldsymbol{\beta}})$} \\
\cline { 3 - 6 } & & RSSE & LAE & RSSE & LAE \\
\hline 0.25 & 0.5 & $\mathbf{0 . 2 0 4 3 4 4}$ & $\mathbf{0 . 2 2 2 2 3 1}$ & $\mathbf{0 . 2 2 9 7 6 1}$ & $\mathbf{0 . 0 9 1 9 4 9}$ \\
\hline & 1 & $\mathbf{0 . 9 8 3 7 1 1}$ & $\mathbf{0 . 8 6 3 5 1 8}$ & $\mathbf{0 . 1 8 9 2 9 6}$ & $\mathbf{0 . 0 9 5 5 4}$ \\
\hline & 2.5 & $\mathbf{0 . 6 8 6 8 2 4}$ & $\mathbf{0 . 9 0 3 7 3 3}$ & $\mathbf{0 . 1 6 2 8 0 6}$ & $\mathbf{0 . 2 2 0 6 3}$ \\
\hline $\mathbf{0 . 7 5}$ & $\mathbf{0 . 5}$ & $\mathbf{0 . 1 1 3 3 8 3}$ & $\mathbf{0 . 1 0 5 0 5 6}$ & $\mathbf{0 . 3 4 6 9 7 4}$ & $\mathbf{0 . 2 5 2 9 3 9}$ \\
\hline & 1 & $\mathbf{0 . 2 2 4 4 8 2}$ & $\mathbf{0 . 2 0 0 1 4 9}$ & $\mathbf{0 . 0 9 5 0 2 9}$ & $\mathbf{0 . 1 4 2 5 1 2}$ \\
\hline & 2.5 & $\mathbf{0 . 4 6 7 1 2 6}$ & $\mathbf{0 . 4 8 3 8 4 7}$ & $\mathbf{0 . 1 7 3 2 7 6}$ & $\mathbf{0 . 1 6 8 8 1 7}$ \\
\hline 1.5 & 0.5 & $\mathbf{0 . 0 3 8 3 2 3}$ & $\mathbf{0 . 0 4 8 3 5 2}$ & $\mathbf{0 . 1 3 7 4 6 2}$ & $\mathbf{0 . 2 0 6 7 7 5}$ \\
\hline & 1 & $\mathbf{0 . 2 3 7 0 8 6}$ & $\mathbf{0 . 2 3 5 5 7 1}$ & $\mathbf{0 . 2 0 9 9 7 1}$ & $\mathbf{0 . 1 9 2 0 9 3}$ \\
\hline & 2.5 & $\mathbf{0 . 4 9 7 8 7 5}$ & $\mathbf{0 . 4 6 9 1 0 1}$ & $\mathbf{0 . 0 9 4 2 0 6}$ & $\mathbf{0 . 1 8 9 2 0 4}$ \\
\hline 2.5 & 0.5 & $\mathbf{0 . 0 4 7 5 8 9}$ & $\mathbf{0 . 0 4 3 9 6 5}$ & $\mathbf{0 . 1 0 9 8 7 3}$ & $\mathbf{0 . 0 9 9 8 9 1}$ \\
\hline & 1 & $\mathbf{0 . 0 6 9 4 0 1}$ & $\mathbf{0 . 0 6 0 3 5 4}$ & $\mathbf{0 . 1 3 3 4 5 1}$ & $\mathbf{0 . 1 3 0 8 1 9}$ \\
\hline & 2.5 & $\mathbf{0 . 1 0 2 8 8}$ & $\mathbf{0 . 1 0 7 9 8 4}$ & $\mathbf{0 . 0 6 7 2 3 2}$ & $\mathbf{0 . 1 7 1 7 8}$ \\
\hline
\end{tabular}

When $\mathrm{n}=50$

\begin{tabular}{|l|l|l|l|l|l|}
\hline \multirow{2}{*}{$\alpha$} & \multirow{3}{*}{$\boldsymbol{\beta}$} & \multicolumn{2}{|l|}{$\operatorname{MAPE}(\widehat{\boldsymbol{\alpha}})$} & \multicolumn{2}{l|}{$\operatorname{MAPE}(\widehat{\boldsymbol{\beta}})$} \\
\cline { 3 - 6 } & & RSSE & LAE & RSSE & LAE \\
\hline 0.25 & 0.5 & $\mathbf{0 . 1 9 2 0 7 4}$ & $\mathbf{0 . 1 7 8 1 7 2}$ & $\mathbf{0 . 2 8 2 6 3}$ & $\mathbf{0 . 2 0 4 7 5 2}$ \\
\hline & 1 & $\mathbf{0 . 7 2 2 1 7 9}$ & $\mathbf{0 . 6 9 1 1 5 7}$ & $\mathbf{0 . 1 1 1 4 3 9}$ & $\mathbf{0 . 0 7 6 9 2 3}$ \\
\hline & 2.5 & 2.43513 & 2.38861 & $\mathbf{0 . 0 7 7 5 1 7}$ & $\mathbf{0 . 0 9 4 2 3 4}$ \\
\hline $\mathbf{0 . 7 5}$ & $\mathbf{0 . 5}$ & $\mathbf{0 . 0 2 8 0 5 3}$ & $\mathbf{0 . 0 2 2 8 0 6}$ & $\mathbf{0 . 1 9 9 1 8 8}$ & $\mathbf{0 . 0 8 7 3 5 1}$ \\
\hline & 1 & $\mathbf{0 . 1 8 2 3 9 6}$ & $\mathbf{0 . 1 9 8 3 3 8}$ & $\mathbf{0 . 2 1 6 0 5 3}$ & $\mathbf{0 . 1 2 7 7 1 1}$ \\
\hline & 2.5 & $\mathbf{0 . 2 0 6 2 5 3}$ & $\mathbf{0 . 2 8 4 8 0 7}$ & $\mathbf{0 . 1 2 2 9 3 5}$ & $\mathbf{0 . 1 2 8 8 2 5}$ \\
\hline 1.5 & 0.5 & $\mathbf{0 . 0 5 1 5 3}$ & $\mathbf{0 . 0 4 1 6 4 6}$ & $\mathbf{0 . 2 2 7 9 1 6}$ & $\mathbf{0 . 0 9 8 3 5}$ \\
\hline
\end{tabular}




\begin{tabular}{|l|l|l|l|l|l|}
\hline & 1 & $\mathbf{0 . 0 8 7 0 7 8}$ & $\mathbf{0 . 0 8 0 4 6 7}$ & $\mathbf{0 . 1 1 6 5 1}$ & $\mathbf{0 . 0 4 1 1 8 9}$ \\
\hline & 2.5 & $\mathbf{0 . 1 6 4 6 8 7}$ & $\mathbf{0 . 1 5 6 6 7 3}$ & $\mathbf{0 . 0 5 4 4 4 9}$ & $\mathbf{0 . 0 3 1 0 1 3}$ \\
\hline 2.5 & 0.5 & $\mathbf{0 . 0 1 8 1 9}$ & $\mathbf{0 . 0 1 8 9 3 3}$ & $\mathbf{0 . 1 1 2 9 7 4}$ & $\mathbf{0 . 0 6 7 8 5 4}$ \\
\hline & 1 & $\mathbf{0 . 0 5 1 7 5 1}$ & $\mathbf{0 . 0 4 8 9 0 4}$ & $\mathbf{0 . 0 4 6 8 3}$ & $\mathbf{0 . 0 4 3 3 9 4}$ \\
\hline & 2.5 & $\mathbf{0 . 0 3 4 0 3 1}$ & $\mathbf{0 . 0 4 4 3 2 3}$ & $\mathbf{0 . 1 4 0 2 7 8}$ & $\mathbf{0 . 1 4 5 1 3 4}$ \\
\hline
\end{tabular}

From table(3) we can make the following comments:

1-The values of MAPE for $\hat{\boldsymbol{\alpha}}$ and $\hat{\beta}$ are vibrating for all values of samples sizes (n).

2- The values of MAPE for $\widehat{\boldsymbol{\alpha}}$ are increasing once time for all values of $\alpha$ and $\beta$ and vibrating another time for all values of $\alpha$ and $\beta$ in lindely approximation bayes method and for Rank sampling set method.

3-Showing that the smallest values of MAPE for $\widehat{\boldsymbol{\alpha}}$ are (0.046884) when $(\alpha=2.5 \quad, \beta=1 \quad, n=10)$ for Lindley approximation method, while MAPE is $(0.038323)$ when $(\alpha=1.5, \beta=0.5$ $, \mathrm{n}=20)$ for Rank sampling set method, and (0.01819) when $(\alpha=2.5, \beta=0.5$ $, \mathrm{n}=50)$ for Lindley approximation method.

4-The values of MAPE for $\hat{\beta}$ are increasing once time, decreasing other time and vibrating another time for all values of $\alpha$ and $\beta$ in Rank sampling set method and Lindley approximation Bayes method.

5-Noting that the smallest values of MAPE for $\hat{\beta}$ is $(0.4002)$ when $(\alpha=2.5$, $\beta=1, n=10)$ for Lindley approximation method, while MAPE is (0.067232) when $(\alpha=2.5, \beta=2.5, \quad n=20)$ for Rank sampling set method ,and (0.031013)when $(\alpha=1.5, \beta=2.5, \mathrm{n}=50)$ for Lindley approximation method.

\section{References:}

1. Balakrishnan N. (1992), "Handbook of the Logistic Distribution",Marcel Dekker, Inc., New York, Basel. Hong. Kong.

2. Plackett, Robin L. (1959). "The Analysis of Life Test Data", Technometrics, 1(1), 9-19.
3. Oliver, F. R. (1964). "Methods of Estimating the Logistic Growth Function," Applied Statistics, 13(2), 57-66.

4. Henrick J.M. and Bovas A.,(1973), "Multivarite logistic distribution", The annals of statistics, 1(3):588-590.

5. Olapade A. K.,(2005) "On negatively skewed extended generalized logistic distribution", kragujevac J.Math,27:175_182

6. Scerri E. and Farrugia R.,(1996),"Wind data evaluation in the Maltese Islands", Renewable Energy ,7(1):109-114.

7. Jones M. C. (2006), "On a Class of Distribution Defined by the Relationship Between Their Density and Distribution Functions",Taylor and francis 36(10):1835_1843.

8. Al-Hajar Z. A.,(2009), "Generating random variates for estimating the parameter of logistic distribution by montecarlo simulation", M.SC. Thesis ,college of science, Al-Nahrain University ,Baghdad.

9. SubrataCh.,Partha J. H. and M. Masoom Ali,(2012), "Anew skew logistic distribution and its properties ",Pak. J. Statist, 28(4):513-524.

10. Douglas A.W., (2004),"An Approach to More Efficient Data Collection",Statist. Sci. 19( 4) : 636-643. 
11. Carlos N. Bouza, (2005),"

Distribution:

Bayesian Sampling using Ranked Estimations", Journal Sets:Concept, Results and Computational statistics \&Data Perspctives",RevistaInestigaciónO peracional, 26(3):275. Analysis, volume 52 Issue 4,January,pages 1873-1883.

12. DebasisK. andRameshwar D. G., (2008),"Generalized Exponential

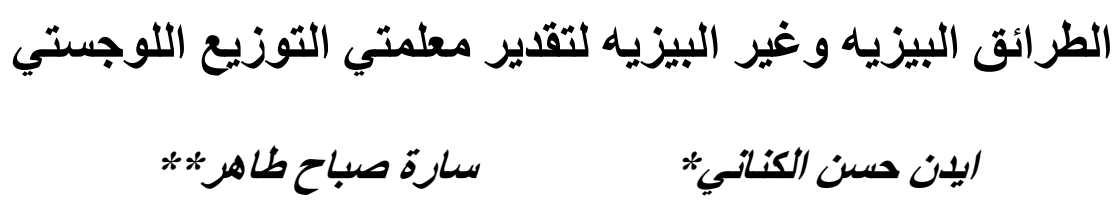

*قسم الرياضيات ،كلية العلوم للبنات ،جامعة بغداد ،بغداد ،العر اق.

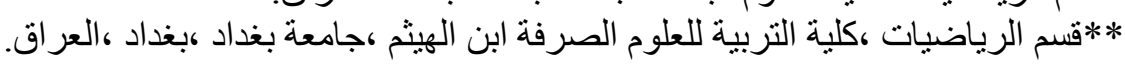

في هذا البحث ناقتشنا مسأله تقدير النقطة للتوزيع اللوجستي ذي المعلمتين باعتماد اسلوب المحاكاة،وقدرنا

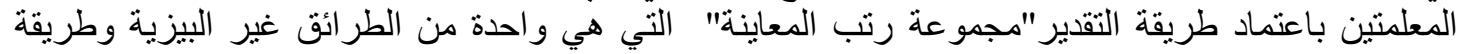

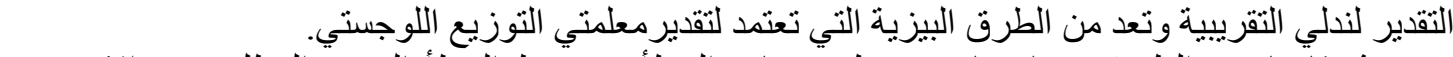

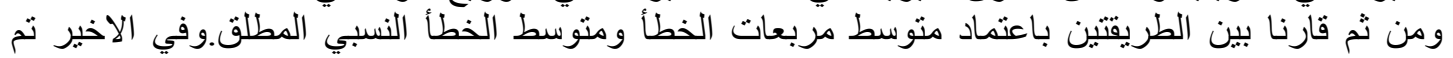

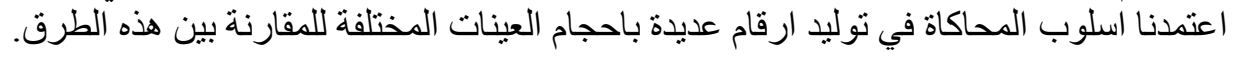

